\title{
Quasi-maximum likelihood estimation of stochastic volatility models
}

\author{
Esther Ruiz \\ Departamento de Estadistica y Econometria. Unicersidad Carlox III. C/Madrid 126-128. \\ 28903 Getafe, Spain
}

\begin{abstract}
Changes in variance or volatility over time can be modelled using stochastic volatility (SV) models. This approach is based on treating the volatility as an unobserved variable, the logarithm of which is modelled as a linear stochastic process, usually an autoregression. This article analyses the asymptotic and finite sample properties of a Quasi-Maximum Likelihood (QML) estimator based on the Kalman filter. The relative efficiency of the QML estimator when compared with estimators based on the Generalized Method of Moments is shown to be quite high for parameter values often found in empirical applications. The QML estimator can still be employed when the SV model is generalized to allow for distributions with heavier tails than the normal. SV models are finally fitted to daily observations on the yen/dollar exchange rate.
\end{abstract}

Key words: Exchange rates; Generalized method of moments; Kalman filter; Quasimaximum likelihood; Stochastic volatility

JEL classification: $\mathrm{C} 22$

The author was at the London School of Economics while working on this paper. I would like to thank A.C. Harvey for many helpful comments on earlier drafts of this paper and for sharing with me many of his ideas on stochastic volatility models. I am also grateful to J. Hidalgo, M. Knott, A. Monfort, D.B. Nelson, D. Peña, N.G. Shephard, R. Zamar, an anonymous referee, and participants at the 1992 Meeting of the Econometric Study Group (Bristol), seminars at the Bank of Spain and CEMFI, 1992 ASSET Meeting (Toulousse), and XVIl Simposio de Análisis Económico (Barcelona). Support from the F.SRC, as part of the project 'Estimation and Diagnostic Testing of Unobserved Components Models', grant no. R000 233574 , is gratefully acknowledged. I am responsible for any errors which may remain in this paper. 


\section{Introduction}

Time series models with heteroscedastic errors are receiving an increasing attention in the econometric literature. These models are specially useful for modelling high-frequency financial time series, such as stock returns and exchange rates. In the simplest set up, the series of interest is a white noise process, with unit variance, multiplied by a factor $\sigma_{t}$, known in the financial literature as volatility. That is,

$$
y_{t}=\sigma_{t} \varepsilon_{t}
$$

There are different ways for modelling changes in volatility over time. Some of the most popular models in the literature are those based on the Autoregressive Conditional Heteroscedasticity (ARCH), inspired by Engle (1982); see Bollerslev et al. (1992) for a detailed review of these models. All ARCH-based models share the property that the distribution of $y_{t}$ conditional on past values of the series, up to and including time $t-1$, is Gaussian with variance $\sigma_{t}^{2}$. Consequently, the volatility is observable at time $t-1$.

Alternatively, the volatility may be treated as an unobserved variable, the logarithm of which is modelled as a linear stochastic process, such as an autoregression. Models of this kind are called stochastic volatility (SV) models; see Taylor (1991) for an excellent review on SV models.

ARCH-type models and SV models have similar statistical properties. However, they are different with respect to the observability of $\sigma_{t}^{2}$ at time $t-1$; see Andersen (1992) for a detailed discussion on this subject.

SV models fit quite naturally into the theoretical framework within which much of the modern finance theory, in relation to option valuation, has been developed; see, for example. Chesney and Scott (1989). Another feature of these models is that they can be naturally generalized to multivariate series as in Harvey et al. (1992). However, they are not conditionally Gaussian and, therefore, their estimation and statistical handling may present some difficulties. The estimation of SV models has usually been carried out by variants of the method of moments. The aim of this article is to analyse the properties of a QuasiMaximum Likelihood (QML) estimator of SV models based on the Kalman filter and proposed independently by Nelson (1988) and Harvey et al. (1992) and compare them with the properties of some estimators based on the method of moments.

The article is organised as follows. Section 2 sets out some of the basic ideas of univariate SV models. In Section 3, the QML estimator is described and its asymptotic and finite-sample properties are found. In Section 4, the asymptotic and finite-sample properties of two estimators based on the method of moments are analysed. It is shown that for parameter values likely to arise in practice when analysing very-high-frequency financial time series, these estimators arc 
less efficient than the QML estimator. Section 5 presents an empirical application using a series of daily yen/dollar exchange rates. The conclusions are given in Section 6.

\section{Stochastic volatility processes}

A simple stationary SV model is given by

$$
\begin{aligned}
& y_{t}=\varepsilon_{t} \exp \left\{h_{t} / 2\right\}, \\
& h_{t}=\gamma+\phi h_{t-1}+\eta_{t}, \quad \eta_{t} \sim \operatorname{NID}\left(0, \sigma_{\eta}^{2}\right),
\end{aligned}
$$

where $h_{t}=\ln \left(\sigma_{t}^{2}\right), \varepsilon_{t}$ is a white noise process with unit variance, generated independently of $\eta_{t}$ and $|\phi|<1$. Working with logarithms ensures that $\sigma_{t}^{2}$ is always positive.

Transforming $y_{t}$ by taking logarithms of the squares, we obtain a linear state space model

$$
\begin{aligned}
& \ln \left(y_{t}^{2}\right)=\mathrm{E}\left(\ln \left(\varepsilon_{t}^{2}\right)\right)+h_{t}+\xi_{t}, \\
& h_{t}=\vartheta+\phi h_{t-1}+\eta_{t},
\end{aligned}
$$

where $\xi_{t}=\ln \left(\varepsilon_{t}^{2}\right)-\mathbf{E}\left(\ln \left(\varepsilon_{t}^{2}\right)\right) . \xi_{t}$ is a non-Gaussian, zero mean, white noise, and its statistical properties depend on the distribution of $\varepsilon_{t}$. From (3) it is possible to observe that, $\ln \left(y_{t}^{2}\right)$ is equivalent to an $\operatorname{ARMA}(1,1)$ process with a nonGaussian noise.

If $\varepsilon_{t} \sim \mathrm{NID}(0,1)$, as it is often assumed in the literature, the mean and variance of $\ln \left(\varepsilon_{\mathrm{f}}^{2}\right)$ are known to be $\psi\left(\frac{1}{2}\right)-\ln \left(\frac{1}{2}\right) \approx-1.27$ and $\pi^{2} / 2$ respectively, where $\psi(\cdot)$ is the Digamma function; see Abramovitz and Stegun (1970).

Some authors also assume that $\varepsilon_{t}$ have a Student- $t$ distribution; see Harvey et al. (1992). Let $\varepsilon_{\mathrm{r}}$ be a $t$-variable written as

$$
\varepsilon_{t}=\zeta_{t} / \kappa_{t}^{1 / 2},
$$

where $\zeta_{t}$ is a standard normal variate and $v \kappa_{t}$ is distributed, independently of $\zeta_{t}$, as a $\chi^{2}$ with $v$ degrees of freedom. Then $\ln \left(\varepsilon_{t}^{2}\right)=\ln \left(\zeta_{t}^{2}\right)-\ln \left(\kappa_{t}\right)$. It follows from results in Abramovitz and Stegun (1970) that, in this case, $E\left[\ln \left(\varepsilon_{t}^{2}\right)\right] \approx$ $-1.27-\psi(v / 2)+\ln (v / 2)$ and $\operatorname{var}\left[\ln \left(\varepsilon_{t}^{2}\right)\right]=\pi^{2} / 2+\psi^{\prime}(v / 2)$, where $\psi^{\prime}(\cdot)$ is the Trigamma function.

The SV model in (2) can be generalised so that $h$ follows any stationary ARMA process. Alternatively, $h_{t}$ can be allowed to follow a random walk. The corresponding SV model is then given by

$$
\begin{aligned}
& y_{t}=\varepsilon_{t} \exp \left\{h_{t} / 2\right\}, \\
& h_{t}=h_{t} \quad 1+h_{t} .
\end{aligned}
$$


In this case, $\ln \left(y_{t}^{2}\right)$ is a random walk plus noise, and the best linear predictor of the current value of $h_{t}$ is an exponential weighted moving average (EWMA) of past values of $\ln \left(y_{t}^{2}\right)$.

\section{Quasi-maximum likelihood estimation}

The QML approach for estimating SV models has been proposed independently by Nelson (1988) and Harvey et al. (1992) and is based on the Kalman filter. This is applied to $\ln \left(y_{t}^{2}\right)$ to obtain one-step-ahead errors and their variances. These are then used to construct a quasi-likelihood function. Because $\ln \left(y_{t}^{2}\right)$ is not Gaussian, the Kalman filter yields minimum mean square linear estimators (MMSLE) of $h_{t}$ and future observations rather than minimum mean square estimators (MMSE).

\subsection{Stationary case}

Consider the SV model (2) with $\varepsilon_{t} \sim \operatorname{NID}(0,1)$. In order to estimate the parameters $\gamma, \phi$, and $\sigma_{\eta}^{2}$, consider the linear state space model in (3), which in this case is given by

$$
\begin{aligned}
& \ln \left(y_{t}^{2}\right)=-1.27+h_{t}+\xi_{t}, \\
& h_{t}=\gamma+\phi h_{t-1}+\eta_{t} .
\end{aligned}
$$

where $\sigma_{\xi}^{2}=\pi^{2} / 2$.

Estimation of model (6) can be carried out by QML by treating $\xi_{t}$ as though it were $\operatorname{NID}\left(0, \pi^{2} / 2\right)$. The Kalman filter may then be used to obtain the prediction error decomposition form of the Gaussian likelihood, which has to be numerically maximised. Notice that model (6) can be written

$$
\begin{aligned}
& y_{t}^{*}=h_{t}+\xi_{t}, \\
& h_{t}=\phi h_{\mathrm{t}-1}+\eta_{t},
\end{aligned}
$$

where $y_{t}^{*}$ is equal to $\ln \left(y_{t}^{2}\right)$ corrected by its mean given by $\gamma^{*}=-1.27+$ $\gamma /(1-\phi)$.

$\gamma^{*}$ can be consistently estimated by the sample mean of $\ln \left(y_{t}^{2}\right)$. Moreover, the sample mean is a QML estimator of $\gamma^{*}$ uncorrelated with the QML estimator of the stochastic part of the model; see Harvey (1989). In what follows, we treat $\gamma^{*}$, or equivalently $\gamma$, as known and concentrate on the estimation of $\Psi=\left(\phi, \sigma_{\eta}^{2}\right)^{\prime}$.

The standard theory for the estimation of unobserved component time series models with nonnormal errors applies to the estimates of $\Psi$. Using the results in Dunsmuir (1979) and since $\eta_{t}$ and $\xi_{t}$ are martingale differences and have finite 
moments up to at least order four, the asymptotic distribution of the QML estimator of $\Psi$, denoted by $\hat{\Psi}$, is given by

$$
\sqrt{T}(\hat{\Psi}-\Psi) \stackrel{\mathrm{d}}{\sim} \mathrm{N}(0, C(\Psi))
$$

where $T$ is the sample size and the analytic expression for $C(\Psi)$ is given in the appendix.

Table 1 shows the finite-sample approximation to the asymptotic standard errors (ASEs), given by $\sqrt{ } C(\Psi) / \sqrt{ } T$, for different parameter values and sample sizes. The finite-sample properties of $\hat{\psi}$ have been studied by carrying out several Monte Carlo experiments. Table 2 shows the results of some of these experiments. The small-sample bias in the QML estimates for $\phi$ is generally of relatively minor order in the present context with a relatively large number of observations. The QML estimates for $\sigma_{\eta}^{2}$ are also slightly biased in finite samples. Both biases tend to increase when $\phi$ decreases or $\sigma_{\eta}^{2}$ decreases. Comparison between the ASEs in Table 1 and the Monte Carlo standard errors shows that for $T=500$ the ASEs of $\hat{\phi}$ and $\hat{\sigma}_{\eta}^{2}$ underestimate the observed standard errors by quite a large amount when $\phi=0.9$ and $\sigma_{\eta}^{2}=0.09$. This may be due to the fact that both parameters are getting close to the boundary of the permissible parameter space. The ASEs of $\hat{\sigma}_{\eta}^{2}$ are, in general, a better approximation to the observed standard errors.

\subsection{Random walk plus noise case}

When $h_{1}$ follows a random walk as in model (5), the Kalman filter approach is still valid if the restriction $\phi=1$ is imposed. The only difference is that the first

Table 1

Asymptotic standard deviations of QML estimator of stationary stochastic variance model

\begin{tabular}{llll}
\hline & $T=500$ & $T=3000$ & $T=6000$ \\
\hline$\phi=0.9$ & 0.0306 & 0.0125 & 0.0088 \\
$\sigma_{n}^{2}=1$ & 0.2890 & 0.1180 & 0.0834 \\
$\phi=0.7$ & 0.1033 & 0.0422 & 0.0298 \\
$\sigma_{n}^{2}=1$ & 0.4802 & 0.1960 & 0.1386 \\
$\phi=0.9$ & 0.0792 & 0.0323 & 0.0229 \\
$\sigma_{n}^{2}=0.09$ & 0.1004 & 0.0410 & 0.0290 \\
$\phi=0.7$ & 0.6761 & 0.2760 & 0.1951 \\
$\sigma_{n}^{2}=-0.09$ & 0.3407 & 0.1391 & 0.0983 \\
\hline
\end{tabular}


Table 2

Monte Carlo experiments on QML estimator of the stationary stochastic variance model: Average value with standard deviations in parentheses $(1000$ replications)

\begin{tabular}{lccc}
\hline & $T=500$ & $T=3000$ & $T=6000$ \\
\hline$=0.9$ & 0.8927 & 0.9004 & 0.9010 \\
$\sigma_{\eta}^{2}=1$ & $(0.0342)$ & $(0.0170)$ & $(0.0150)$ \\
& 1.0276 & 0.9942 & 0.9964 \\
$\phi=0.7$ & $(0.3117)$ & $(0.1207)$ & $(0.0913)$ \\
& 0.6840 & 0.6982 & 0.6979 \\
$\sigma_{\eta}^{2}=1$ & $(0.1081)$ & $(0.0434)$ & $(0.0230)$ \\
& 1.0612 & 1.0030 & 1.0076 \\
$\phi=0.9$ & $(0.5015)$ & $(0.1935)$ & $(0.1400)$ \\
& 0.7464 & 0.8932 & 0.8968 \\
$\sigma_{\eta}^{2}=0.09$ & $(0.2691)$ & $(0.0406)$ & $(0.0254)$ \\
& 0.2178 & 0.0996 & 0.0949 \\
$\phi=0.7$ & $(0.3314)$ & $(0.0528)$ & $(0.0329)$ \\
$\sigma_{\eta}^{2}=0.09$ & 0.3688 & 0.5362 & 0.6138 \\
& $(0.2857)$ & $(0.2841)$ & $(0.2314)$ \\
\hline
\end{tabular}

observation is used to initialise the Kalman filter, whereas when $\phi<1$, the unconditional distribution of $h_{t}$ is available at $t=0$.

Assuming that $\varepsilon_{t} \sim \operatorname{NID}(0,1)$, consider the following state space model:

$$
\begin{aligned}
& \ln \left(y_{t}^{2}\right)=-1.27+h_{t}+\xi_{t}, \\
& h_{t}=h_{t-1}+\eta_{t},
\end{aligned}
$$

where $\sigma_{\xi}^{2}=\pi^{2} / 2$. In this case, the model contains only one unknown parameter, $\sigma_{\eta}^{2}$. Denote by $\hat{\sigma}_{\eta}^{2}$ the QML estimator of $\sigma_{\eta}^{2}$. The asymptotic distribution of $\sqrt{ } T\left(\hat{\sigma}_{\eta}^{2}-\sigma_{\eta}^{2}\right)$ is normal with zero mean and asymptotic variance $C\left(\sigma_{\eta}^{2}\right)$. The analytic expression for $C\left(\sigma_{\eta}^{2}\right)$ appears in the Appendix.

Table 3 shows the approximation in finite-sample sizes to the ASEs of $\hat{\sigma}_{\eta}^{2}$, for different values of $\sigma_{\eta}^{2}$ and different sample sizes.

The results of several Monte Carlo experiments, carried out to analyse the finite-sample properties of $\hat{\sigma}_{\eta}^{2}$, appear in Table 4 . The QML estimates of $\sigma_{\eta}^{2}$ show a slight finite-sample bias which tends to increase when $\sigma_{\eta}^{2}$ decreases. Moreover, comparing Tables 3 and 4 , one observes that, for small values of $\sigma_{\eta}^{2}$, the ASEs underestimate the observed standard errors. The problem may be that, when $\sigma_{\eta}^{2}$ is close to zero, the distribution of $\hat{\sigma}_{\eta}^{2}$ may be some way from normality; see 
Table 3

Standard deviation of QML estimator of random walk volatility model

\begin{tabular}{llll}
\hline$\sigma_{\eta}^{2}$ & $T=500$ & $T=3000$ & $T=6000$ \\
\hline 0.09 & 0.03196 & 0.01305 & 0.00922 \\
0.01 & 0.00603 & 0.00246 & 0.00174 \\
0.0009 & 0.00098 & 0.00040 & 0.00028 \\
0.0001 & 0.00019 & 0.00008 & 0.00005 \\
\hline
\end{tabular}

Table 4

Monte Carlo experiments on QML estimator of the random walk volatility model: Average value with standard deviations in parentheses ( 1000 replications)

\begin{tabular}{lccc}
\hline$\sigma_{\eta}^{2}$ & $T=500$ & $T=3000$ & $T=6000$ \\
\hline 0.09 & 0.09028 & 0.08949 & 0.09005 \\
& $(0.03356)$ & $(0.01269)$ & $(0.00903)$ \\
0.01 & 0.01015 & 0.00996 & 0.01002 \\
& $(0.00689)$ & $(0.00247)$ & $(0.00175)$ \\
0.0009 & 0.00101 & 0.00089 & 0.00091 \\
& $(0.00148)$ & $(0.00042)$ & $(0.00028)$ \\
0.0001 & 0.00028 & 0.00010 & 0.00010 \\
& $(0.00083)$ & $(0.00009)$ & $(0.00006)$ \\
\hline
\end{tabular}

Harvey (1989). For relatively large values of $\sigma_{\eta}^{2}$, the ASEs are good approximations to the finite-sample standard deviations.

\subsection{Heavy-tailed distributions}

When $\delta_{t}$ is a $t$-variable as given by (4) and $h_{t}$ is stationary, consider the state space model in (7) with $\gamma^{*}=-1.27-\psi(v / 2)+\log (v / 2)-\gamma /(1-\phi)$ and $\sigma_{\xi}^{2}=\pi^{2} / 2+\psi^{\prime}(v / 2)$. Then, the model can be estimated by estimating $\sigma_{\xi}^{2}, \gamma^{*}, \phi$, and $\sigma_{\eta}^{2}$ with the restriction $\sigma_{\varepsilon}^{2} \geqslant \pi^{2} / 2$. The estimated variance of $\xi_{t}$ implies a value of $v$. As in the Gaussian case, $\gamma^{*}$ can be estimated by the sample mean and therefore we concentrate on the estimation of $\psi^{*}=\left(\sigma_{z}^{2}, \phi, \sigma_{\eta}^{2}\right)^{\prime}$. Estimates of $\Psi^{*}$ can be obtained by treating $\vec{\zeta}_{r}$ as though it were NID $\left(0, \sigma_{c}^{2}\right)$ and maximising the resulting quasi-likelihood function. In this case, the asymptotic distribution of the QML estimator of $\psi *$ is given by

$$
\sqrt{T\left(\hat{\Psi}^{*}-\Psi^{*}\right) \stackrel{\mathrm{d}}{\sim} \mathrm{N}\left(0, C\left(\Psi^{*}\right)\right) .}
$$

The elements of $C\left(\Psi^{*}\right)$ appear in the Appendix. 
It is also possible to estimate SV models by assuming that $y$ is known. In this case, there is no need to estimate $\sigma_{\xi}^{2}$. Surprisingly, for the typical parameter values often found in empirical applications, the estimates of $\phi$ and $\sigma_{\eta}^{2}$ are less efficient in this case. Table 5 reports values of ASEs of the QML estimators of $\Psi=\left(\phi, \sigma_{n}^{2}\right)^{\prime}$ obtained by assuming that $v$ is known and when this parameter is estimated. It can be observed that the loss in efficiency is greater for $\hat{\sigma}_{n}^{2}$ than for $\hat{\phi}$. For the parameter values considered in Table 5, the loss in efficiency for $\hat{\phi}$ can be shown to be quite small. However, for $\hat{\sigma}_{\eta}^{2}$ the loss could be as big as $3 \%$ in some cases. This result seems counterintuitive at first sight. However, since the proposed estimators are QML and not maximum likelihood estimators, they are not fully efficient. Consequently, it may be possible that the QML estimator of $\sigma_{n}^{2}$ when both variances are estimated is more efficient than when $\sigma_{\xi}^{2}$ is known.

With respect to hypothesis testing, an interesting hypothesis to test is whether $\varepsilon_{t}$ is normal, i.e., $\boldsymbol{H}_{0}: \sigma_{b}^{2}=\pi^{2} / 2$. One possible test statistic could be a quasiLikelihood Ratio (LR) test. Since under the null hypothesis, $\sigma_{\xi}^{2}$ is on the

Table 5

Asymptotic standard deviations with Student-t disturbances and relative efficiency computed as the ratio of standard deviations

\begin{tabular}{llll}
\hline & Estimating $v$ & $v$ known & Efficiency loss \\
\hline (a) Stationarity & & & \\
$y=6$ & & 0.6708 & 1.0112 \\
$\phi=0.95$ & 0.6634 & 1.3847 & 1.0199 \\
$\sigma_{\eta}^{2}=0.09$ & 1.3577 & & 1.0139 \\
$y=10$ & & 0.6647 & 1.0246 \\
$\phi=0.95$ & 0.6556 & 1.3670 & 1.0139 \\
$\sigma_{\eta}^{2}=0.09$ & 1.3342 & & 1.0221 \\
$y=6$ & & 2.2785 & 1.0024 \\
$\phi=0.95$ & 2.2472 & 0.6792 & 1.0111 \\
$\sigma_{\eta}^{2}=0.01$ & 0.6645 & & \\
$y=6$ & & 0.1650 & 1.0280 \\
$\phi=0.99$ & 0.1646 & 0.8367 & 1.0088 \\
$\sigma_{\eta}^{2}=0.09$ & 0.8275 & & 1.0041 \\
\hline$(b)$ Random walk & & & \\
$y=6$ & & 0.7384 & \\
$\sigma_{\eta}^{2}=0.09$ & 0.7183 & 0.1379 & \\
$v=6$ & & 0.0243 & \\
$\sigma_{\eta}^{2}=0.01$ & 0.1367 & & \\
$y=6$ & 0.0242 & & \\
$\sigma_{\eta}^{2}=0.001$ & & & \\
\hline
\end{tabular}


boundary of the admissible parameter space, the distribution of the LR test is given by

$$
\mathbf{L R} \sim \frac{1}{2} \chi_{0}^{2}+\frac{1}{2} \chi_{1}^{2}
$$

where $\chi_{0}^{2}$ is a degenerate distribution with all its mass at the origin; see Harvey (1989). The size of the LR test can therefore be set appropriately simply by using the $2 \alpha$, rather than the $\alpha$, significance point of a $\chi_{1}^{2}$ distribution for a test of size $\alpha$. For example, for $\alpha=5 \%$, the corresponding critical value for the quasi-LR test statistic is 2.71. This is the value found by Bollerslev (1987) by carrying out Monte Carlo experiments.

Alternatively, by estimating $\sigma_{\xi}^{2}$ as an unrestricted parameter (i.e., without imposing the lower bound $\pi^{2} / 2$ ), it is possible to allow $\varepsilon_{t}$ to have a distribution with kurtosis which could be smaller or bigger than the normal kurtosis. For example, $\varepsilon_{\mathrm{t}}$ may have a generalized error distribution (GED); see Nelson (1988). The reason why the GED family of distributions could be an attractive alternative is because it includes the normal as an special case, and also includes distributions with thinner and fatter tails than the normal.

Finally, when $\varepsilon_{t}$ is a $t$-variate and $h_{t}$ follows a random walk as in (5), the corresponding SV model can be estimated by estimating $\Psi^{+}=\left(\sigma_{\xi}^{2} \sigma_{\eta}^{2}\right)^{\prime}$ in the appropriate linear state space model. The asymptotic distribution of $\sqrt{ } T\left(\hat{\Psi}^{\dagger}-\Psi^{\dagger}\right)$ is also normal with zero mean and variances and covariances matrix, $C\left(\Psi^{\dagger}\right)$, given in the Appendix.

\section{Estimators based on the method of moments principle}

The estimation of SV models has usually been carried out by variants of the method of moments. These methods have the difficulty that their efficiency depends on the choice of moments. In this section, we compare the asymptotic properties of the QML estimator of SV models with two estimators based on the method of moments principle. In what follows we will assume that $\varepsilon_{t} \sim \operatorname{NID}(0,1)$.

\subsection{Estimator based on the sample variance}

Consider the SV model in (5) with $h_{t}$ following a random walk. The corresponding linear state space form is given by (8).

The stationary form of $y_{t}^{+}=\ln \left(y_{t}^{2}\right)$ is given by $\Delta y_{t}^{\dagger}=\eta_{t}+\Delta \xi_{t}$.

Given that $\eta_{t}$ and $\xi_{t}$ are mutually uncorrelated and $\sigma_{\xi}^{2}=\pi^{2} / 2$, the variance of

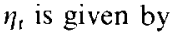

$$
\sigma_{\eta}^{2}=\sigma_{A y}^{2} \dagger-\pi^{2}
$$


A method of moments estimator of $\sigma_{\eta}^{2}$ is then given by

$$
\tilde{\sigma}_{\eta}^{2}=\tilde{\sigma}_{j y}^{2} \dagger-\pi^{2}
$$

where $\tilde{\sigma}_{1 y}^{2} \dagger$ is the sample variance of $\Delta \ln \left(y_{t}^{2}\right)$.

If $\sigma_{n}^{2}>0$, then $T\left(\tilde{\sigma}_{n}^{2}-\sigma_{n}^{2}\right)$ has an asymptotic normal distribution with zero mean and variance $C_{2}=2\left[\left(\sigma_{n}^{2}+\pi^{2}\right)^{2}+\pi^{4}\right]$.

Table 6 shows the ASEs of $\hat{\sigma}_{\eta}^{2}$ and $\sqrt{C_{2}} / \sqrt{T}$, together with the square root of the relative efficiency of $\tilde{\sigma}_{\eta}^{2}$ with respect to $\hat{\sigma}_{\eta}^{2}$. It is possible to observe that, for the parameter values considered, the efficiency of the method of moments estimator compared with the QML estimator is exceptionally low.

\subsection{Generalized method of moments estimator}

Melino and Turnbull (1990) propose an estimator of SV models based on the Generalized Method of Moments (GMM) principle. Consider the stationary SV model in (2) with $\varepsilon_{t} \sim \operatorname{NID}(0,1)$. The functions used by Melino and Turnbull to compute the GMM estimator of model (2) are given by

$$
\begin{aligned}
& y_{t}^{2}-\mathrm{E}\left(y_{t}^{2}\right), \\
& y_{t}^{4}-\mathrm{E}\left(y_{t}^{4}\right), \\
& \left|y_{t}\right|-\mathrm{E}\left(\left|y_{t}\right|\right), \\
& \left|y_{t}^{3}\right|-\mathrm{E}\left(i y_{t}^{3} \mid\right), \\
& y_{t}^{2} y_{t-\tau}^{2}-\mathrm{E}\left(y_{t}^{2} y_{t-\tau}^{2}\right), \quad \tau=1, \ldots, 10 . \\
& \left|y_{t} y_{t-\tau}\right|-\mathrm{E}\left|y_{t} y_{t-\tau}\right|, \quad \tau=1, \ldots, 10 .
\end{aligned}
$$

Denotc by $\tilde{\Psi}$ the GMM estimator of $\Psi$. Under suitable conditions, Hansen (1982) shows that $\sqrt{T(\tilde{\Psi}-\Psi)}$ is asymptotically distributed as a normal variable with zero mean and covariance matrix given by $W=\left(D^{\prime} V^{-1} D\right)^{-1}$, where $D^{\prime}=$ plim $(\partial g(\Psi) / \hat{c} \Psi), g(\Psi)=(1 / T) \Sigma g_{t}, g_{t}$ denote a vector whose components are functions of $y_{1}$ drawn from $(10)$, and $V=\mathrm{E}\left(g_{r} g_{t}^{\prime}\right)$.

Table 6

Asymptotic standard deviation of QML, and method of moments (MM) estimators of random walk volatility model

\begin{tabular}{llll}
\hline$\sigma_{n}^{2}$ & QML & MM & (Rel. eff.) (12 $^{1 / 2}$ \\
\hline 0.09 & 0.7763 & 19.8294 & 0.0391 \\
0.01 & 0.1391 & 19.7492 & 0.0070 \\
0.0009 & 0.0222 & 19.7401 & 0.0011 \\
0.0001 & 0.0042 & 19.7393 & 0.0002 \\
\hline
\end{tabular}


The derivatives needed to compute $D$ have been derived by Vetzal (1991). The moments involved in the construction of $V$ can be derived using the moments of $y_{t}$ given in an Appendix to Melino and Turnbull (1990), kindly supplied to us by the authors.

Table 7 shows the ASEs of $\tilde{\psi}$ together with the ASEs of the QML estimator. Table 7 also shows the square root of the relative efficiency of the GMM estimator with respect to the QML estimator. Comparing the ASEs of both estimators, it is possible to observe that, in terms of efficiency, the QML estimation method performs better when $\phi$ is close to one and $\sigma_{\eta}^{2}$ is relatively big. On the other hand, when $\sigma_{\eta}^{2}$ and $\phi$ are small, the GMM estimator is more efficient. This result could be expected, because when $\sigma_{\eta}^{2}$ and $\phi$ are small, the variance of the log-volatility process, given by $\sigma_{\eta}^{2} /\left(1-\phi^{2}\right)$, is very small in relation to the variance of $\xi_{1}$. Therefore, the transformation $\ln \left(y_{t}^{2}\right)$ is dominated by $\xi_{t}$, and the approximation to normality used by the Kalman filter is very poor. Also, in these circumstances, the process $y_{t}$ is approximately normal. with the excess kurtosis being small. As a consequence, the sample moments are reliable estimators of the population moments, and the GMM estimator performs quite well.

However, in most empirical applications with very-high-frequency financial time series (hourly or daily), it has been observed that the parameter $\phi$ is very close or exactly one. The estimated values for $\sigma_{\eta}^{2}$ are usually between 0.01 and 2.77; see Taylor (1991). With this range of parameter values, there is little doubt

Table 7

Asymptotic standard deviations of QML and GMM estimators

\begin{tabular}{|c|c|c|c|}
\hline & QML & GMM & $(\text { Re], eff. })^{1 / 2}$ \\
\hline$\phi=0.9$ & 0.68 & 12203.65 & 0.00 \\
\hline$\sigma_{n}^{2}=1$ & 6.46 & 28903.37 & 0.00 \\
\hline$\phi=0.7$ & 2.31 & 29.17 & 0.08 \\
\hline$\sigma_{y}^{2}-1$ & 10.74 & 20.13 & 0.53 \\
\hline$\phi=0.9$ & 1.77 & 3.77 & 0.47 \\
\hline$\sigma_{\eta}^{2}=0.09$ & 2.24 & 0.86 & 2.60 \\
\hline$\phi-0.7$ & 15.12 & 13.94 & 1.08 \\
\hline$\sigma_{\eta}^{2}=0.09$ & 7.62 & 1.02 & 7.47 \\
\hline$\phi=0.95$ & 0.92 & 3.00 & 0.31 \\
\hline$\sigma_{\eta}^{2}=0.04$ & 0.95 & 0.61 & 1.56 \\
\hline$\phi=0.97$ & 0.47 & 3.54 & 0.13 \\
\hline$\sigma_{\eta}^{2}=0.04$ & 0.69 & 1.17 & 0.59 \\
\hline$\phi=0.99$ & 0.18 & 74.36 & 0.00 \\
\hline$\sigma_{\eta}^{2}=0.04$ & 0.47 & 73.98 & 0.01 \\
\hline
\end{tabular}


about the better performance of the QML estimator. Further, using the GMM estimator in these circumstances could lead to huge losses in efficiency. Observe, for example, the case when $\sigma_{\eta}^{2}=1$ and $\phi=0.9$, in Table 7 . The extremely big standard deviations in this case may be due to the fact that the excess kurtosis of $y_{t}$ is big and, therefore, the sample moments are not reliable estimators of the population moments.

\section{An empirical example}

In this section, we illustrate the QML estimation method by fitting SV models to the yen/dollar exchange rate. The data consist of daily observations of weekdays close exchange rates from $1 / 10 / 81$ to $28 / 6 / 85$, giving $T=946$ (this data was also used in the empirical application reported in Harvey et al.. 1992). The analysed series is the first differences of the logarithms of the spot price, i.c., the rates of return. For convenience, the rates of return have been centered about the sample mean prior to analysis.

Table 8

Empirical estimates of SV models using daily yen/dollar exchange rates with asymplotic standard deviations in parentheses

(a) Stationary SV model

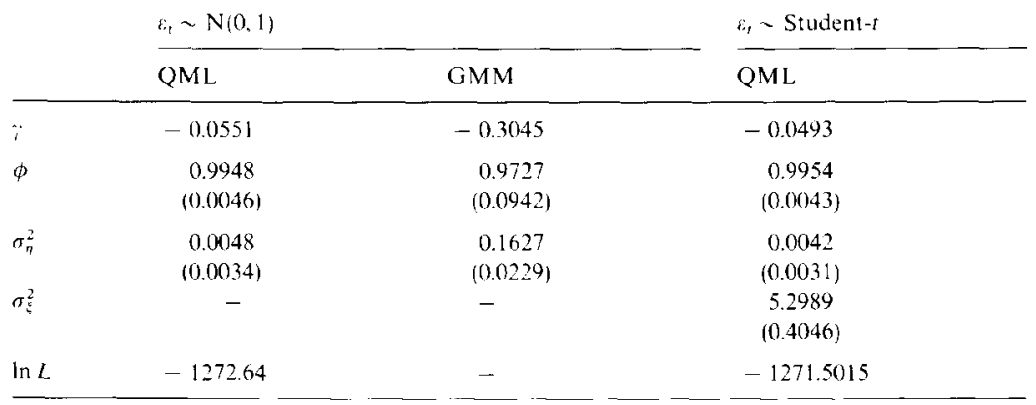

(b) Random walk volatility model

\begin{tabular}{|c|c|c|c|}
\hline & \multicolumn{2}{|l|}{$n_{i} \sim \mathbf{N}(0,1)$} & \multirow{2}{*}{$\frac{t_{t} \sim \text { Student }-t}{\text { QML }}$} \\
\hline & QML & $\mathrm{MM}$ & \\
\hline$\sigma_{n}^{2}$ & $\begin{array}{c}0.0034 \\
(0.0019)\end{array}$ & $\begin{array}{c}0.7649 \\
(0.6671)\end{array}$ & $\begin{array}{c}0.0030 \\
(0.0018)\end{array}$ \\
\hline$\sigma_{\zeta}^{2}$ & - & -- & $\begin{array}{c}5.3039 \\
(0.4038)\end{array}$ \\
\hline $\log t$ & -1273.64 & - & -1272.44 \\
\hline
\end{tabular}


The Box-Ljung statistic for the data, $y_{t}$, based on ten lags, is 16.92 , and therefore not significant at the 5\% significance level. However, the Box-Ljung statistic for $y_{t}^{2}$ is 109.79 , a highly significant value. Therefore, the dynamic properties of the yen/dollar exchange rate show up in the squares and not in the level of the series.

First, the stationary SV model in (2) is fitted to $y_{t}$. Table 8 shows the QML and GMM estimates when $\varepsilon_{1}$ is assumed to be a standard normal variate. Both estimates of the autoregressive parameter imply persistence of the volatility and, as we have previously seen, in this circumstances the efficiency of the GMM method can be very low relative to the efficiency of the QML method. Therefore, we only consider the QML estimates.

As the QML estimate of the autoregressive parameter is very close to one, we estimate the random walk specification in (4). Once more we estimate by QML and using the method of moments (MM) estimator described in Section 4.1. The efficiency of the MM estimator is extremely low relative to the QML estimator. Moreover, the MM estimate is extremely big and not very reliable. Therefore, we concentrate on the QML estimates.

The random walk specification fits almost as well as the stationary SV specification. The Box-Ljung statistic of the innovations for ten lags has a value of 8.45 , giving no indication of residual serial correlation. Fig. 1 shows the

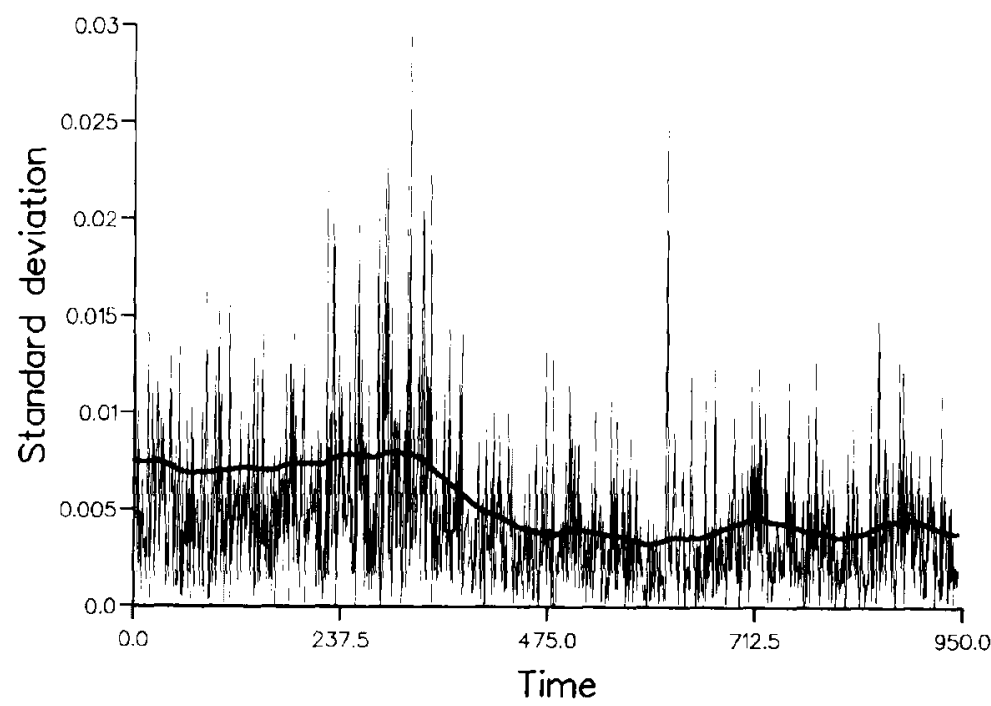

Fig. 1. Absolute value of first differences of logged yen/dollar exchange rate and smoothed estimates of volatility 
absolute values, $\left|y_{t}\right|$, together with the estimated volatility, $\exp \left\{0.5 \tilde{h}_{t T}\right\}$, where $\tilde{h}_{r / T}$ is the MMSLE of the log-volatility level, $h_{1}$, as given by a smoothing algorithm.

Next, the normality assumption for $\varepsilon_{t}$ is relaxed, and the variance of $\ln \left(\varepsilon_{t}^{2}\right), \sigma_{\varepsilon}^{2}$, is estimated as another parameter which is restricted to be greater than or equal to $\pi^{2} / 2$. The estimation results also appear in Table 8 .

The number of degrees of freedom implied by $\hat{\sigma}_{\xi}^{2}$ in the random walk case is 6.35. The $L R$ test statistic takes a value of 2.4 . The critical value for $\alpha=10 \%$ is 1.642. Therefore, we reject normality at the $10 \%$ significance level. The evidence against normality is very weak but, as wc have previously seen, the cfficiency of $\hat{\sigma}_{\eta}^{2}$ is greater if we also estimate $\sigma_{\xi}^{2}$. Consequently, we maintain as our final estimate $\hat{\sigma}_{\eta}^{2}=0.0030$. Monte Carlo experiments carried out for these parameter values have shown that in this case, the ASE is a good approximation to the finite-sample standard error.

\section{Conclusions}

The QML estimator of the parameters of SV models is easy to implement and has good finite-sample properties. It is shown that for the parameter values often found in cmpirical analysis of high-frequency financial time serics, the QML estimator outperforms in terms of efficiency some estimators based on the GMM principle.

The extension to heavy-tailed distributions can be carried out very easily using the $t$-distribution. In this case, the estimation of SV models can be carried out by assuming the number of degrees of freedom, $v$, is known or estimating it as another parameter. When comparing the asymptotic variances of the QML estimators obtained by assuming that $v$ is known or when it is estimated, we found the somewhat counterintuitive result that there is a slight loss in efficiency when estimating $\sigma_{\eta}^{2}$ in the former case. This result may be due to the fact that since our estimators are QML, they are not fully efficient. In any case, given that assuming an incorrect value of $v$ leads to an inconsistent estimate of $\sigma_{\eta}^{2}$, there seems to be no reason for making assumptions about the distribution of $\varepsilon_{t}$.

\section{Appendix}

Asymptotic variance and covariance matrices of the QML estimator of some stochastic volatility models

The derivation of the analytic expressions of all the asymptotic variance and covariance matrices in this article has been carried out, using the results in Dunsmuir (1979), in an appendix which is available upon request. 
The asymptotic variance and covariance matrix of the QML estimator of SV models is given by $C(\Psi)=2 A^{1}+A^{-1} B A^{-1}$, with

$$
A=\frac{1}{2 \pi} \int_{-\pi}^{\pi} \frac{\partial \log g(\lambda) \partial \log g(\lambda)}{\partial \Psi} \frac{\mathrm{d} i}{\partial \Psi^{\prime}}
$$

where $\Psi$ is the vector of parameters to be estimated, $g(\hat{\lambda})$ is the spectral generating function of the stationary form of $\log y_{t}^{2}$, and

$$
B_{i j}=\kappa\left[\frac{1}{2 \pi} \int_{-\pi}^{\pi} k^{*}(\lambda) \frac{\partial g^{-1}(\lambda)}{\partial \Psi_{i}} k(\lambda) \mathrm{d} \lambda\right]_{22}\left[\frac{1}{2 \pi} \int_{-\pi}^{\pi} k^{*}(\lambda) \frac{\partial g^{-1}(\lambda)}{\partial \Psi_{j}} k(\lambda) \mathrm{d} \lambda\right]_{22},
$$

where $k$ is the fourth cumulant of $\log \varepsilon_{t}^{2}, k(\lambda)=\sum_{0}^{\infty} D_{j} e^{i \lambda j}, D_{j}$ is the $1 \times 2$ vector of coefficients corresponding to $\left(\eta_{t} \xi_{l}\right)$ in the stationary representation of $\log y_{l}^{2}$. $k^{*}(\lambda)$ is the transpose conjugate of $k(\lambda)$, and $[M]_{u w}$, denotes the $\{u$, th element of matrix $M$.

(a) Stationary $S V$ model with Gaussian errors

$A$ consists of the following elements:

$$
\begin{aligned}
a_{11}= & 4\left[\frac{1}{2\left(1-\phi^{2}\right)}+\frac{\phi^{2} a}{\left(a^{2}-b^{2}\right)^{3 / 2}}+\frac{1}{b^{2}}\left(1+\frac{a^{3}}{\left(a^{2}-b^{2}\right)^{3 / 2}}-\frac{2 a}{\left(a^{2}-b^{2}\right)^{1 / 2}}\right)\right. \\
& +\frac{2 \phi b}{\left(a^{2}-b^{2}\right)^{3 / 2}}-\frac{1}{q}+\frac{2}{q}\left(\frac{\phi^{2}}{\left(a^{2}-b^{2}\right)^{1 / 2}}+\frac{a}{b^{2}}\left(\frac{a}{\left(a^{2}-b^{2}\right)^{1 / 2}}-1\right)\right. \\
& \left.\left.+\left(1-\frac{a}{\left(a^{2}\right.} \frac{a}{\left.-b^{2}\right)^{1 / 2}}\right)\right)\right] . \\
a_{12}= & \frac{4}{\pi^{2}}\left[\frac{1}{q}\left(\frac{\phi}{\left(a^{2}-b^{2}\right)^{1 / 2}}-\frac{1}{b}\left(1-\frac{a}{\left(a^{2}-b^{2}\right)^{1 / 2}}\right)\right)\right. \\
& \left.+\frac{\phi a}{\left(a^{2}-b^{2}\right)^{3 / 2}}+\frac{b}{\left(a^{2}-b^{2}\right)^{3 / 2}}\right], \\
a_{22}= & \frac{4 a}{\pi^{4}\left(a^{2}-b^{2}\right)^{3 / 2}},
\end{aligned}
$$

where $a=q+1+\phi^{2}, b=-2 \phi$, and $q=2 \sigma_{\eta}^{2} / \pi^{2}$. 
$B$ is given by

$$
\begin{aligned}
B= & \frac{16}{\left(a^{2}-b^{2}\right)^{3}} \\
& \times\left[\begin{array}{cc}
q^{2}(\phi a+b)^{2} & \frac{-q\left(\left(1+\frac{\left.\left.\phi^{2}\right) a+2 \phi b\right)(\phi a+b)}{\pi^{2}}\right.\right.}{-q\left(\left(1+\phi^{2}\right) a+2 \phi b\right)(\phi a+b)} \\
\frac{\pi^{2}}{2} & \frac{\left.\left(1+\phi^{2}\right) a+2 \phi b\right)^{2}}{\pi^{4}}
\end{array}\right] .
\end{aligned}
$$

(b) Random walk colatility model with Gaussian errors

$$
C\left(\sigma_{\eta}^{2}\right)=\frac{2}{\sigma_{n}^{2}}\left[\left(\sigma_{\eta}^{4}+2 \sigma_{\eta}^{2} \pi^{2}\right)^{3 / 2}+\frac{2 \pi^{4} \sigma_{\eta}^{4}}{\sigma_{\eta}^{2}+2 \pi^{2}}\right] .
$$

(c) Stationary SV model with Student-t errors

$A$ consists of the following elements:

$$
\begin{aligned}
a_{11}= & \frac{1}{\sigma^{4}}\left[\frac{\left(1+\phi^{2}\right)^{2} a}{\left(a^{2}-b^{2}\right)^{3 / 2}}+1+\frac{a^{3}}{\left(a^{2}-b^{2}\right)^{3 / 2}}-\frac{2 a}{\left(a^{2}-b^{2}\right)^{1 / 2}}-\frac{8 \phi^{2}\left(1+\phi^{2}\right)}{\left(a^{2}-b^{2}\right)^{3 / 2}}\right], \\
a_{12}= & -\frac{2 q}{\sigma^{2}}\left[\frac{\phi a+b}{\left(a^{2}-b^{2}\right)^{3 / 2}}\right], \\
a_{13}= & \frac{1}{\sigma^{4}}\left[\frac{1+q+\phi^{2}(q-2}{\left(a^{2}-b^{2}\right)^{3 / 2}}-\frac{\left.\phi^{2}\right)}{\left(a^{3}\right.}\right], \\
a_{22}= & 4\left[\frac{1}{2\left(1-\phi^{2}\right)}+\frac{\phi^{2} a}{\left(a^{2}-b^{2}\right)^{3 / 2}}+\frac{1}{b^{2}}\left(1+\frac{a^{3}}{\left(a^{2}-b^{2}\right)^{3 / 2}}-\frac{2 a}{\left(a^{2}-b^{2}\right)^{1 / 2}}\right)\right. \\
& +\frac{2 \phi b}{\left(a^{2}-b^{2}\right)^{3 / 2}}-\frac{1}{q}+\frac{2}{q}\left(\frac{\phi^{2}}{\left(a^{2}-b^{2}\right)^{1 / 2}}+\frac{a}{b^{2}}\left(\frac{a}{\left(a^{2}-b^{2}\right)^{1 / 2}}-1\right)\right. \\
& \left.\left.+\left(1-\frac{a}{\left(a^{2}-b^{2}\right)^{1 / 2}}\right)\right)\right], \\
a_{23}= & \frac{2}{\sigma^{2}}\left[\frac{1}{q\left(\frac{a^{2}}{\left(a^{2}-b^{2}\right)^{1 / 2}}-\frac{1}{b}\left(1-\frac{a}{\left(a^{2}-b^{2}\right)^{1 / 2}}\right)\right)}\right. \\
& +\frac{\phi a}{\left(a^{2}-b^{2}\right)^{3 / 2}}+\frac{b}{\left(a^{2}-\overline{\left.b^{2}\right)^{3 / 2}}\right],} \\
a_{33}= & \frac{a}{\sigma^{4}\left(a^{2}-b^{2}\right)^{3 / 2}} .
\end{aligned}
$$


$B$ is given by

$$
B=\frac{\pi^{4}+\psi^{(3)}(v / 2)}{\sigma^{4}\left(a^{2}-b^{2}\right)^{3}} b_{1} b_{1}^{\prime}
$$

where $\sigma^{2}=\pi^{2} / 2+\psi^{\prime}(v / 2), \psi^{(3)}(\cdot)$ is the Tetragamma function and

$$
b_{1}=\left[\begin{array}{c}
\frac{\left(1+\phi^{2}\right) a+\left(a^{2}-b^{2}\right)^{3 / 2}-a^{3}+2 a b^{2}+4 \phi b\left(1+\phi^{2}\right)}{\sigma^{2}} \\
-2 q(\phi a+b) \\
\frac{\left(1+\phi^{2}\right) a+2 \phi b}{\sigma^{2}}
\end{array}\right] .
$$

(d) Random walk volatility model with Student-t errors

$A^{-1}$ is given by

$$
A^{-1}=\frac{\sigma^{4}}{(q+2)-(q(q+4))^{1 / 2}}\left[\begin{array}{cc}
(q+2) & -2 q \\
-2 q & (q(q+4))^{3 / 2}-q^{2}(q+6)
\end{array}\right] .
$$

$B$ is given by

$$
\begin{aligned}
B= & \frac{4\left(\pi^{4}+\psi^{(3)}(v / 2)\right.}{\sigma^{8}(q(q+4))^{3}} \\
& \times\left[\begin{array}{lc}
0.25\left(\left(q^{2}+4 q\right)^{3 / 2}-q^{2}(q+6)\right)^{2} & 0.59\left(\left(q^{2}+4 q\right)^{3 / 2}-q^{2}(q+6)\right) \\
0.59\left(\left(q^{2}+4 q\right)^{3 / 2}-q^{2}(q+6)\right) & q^{2}
\end{array}\right]
\end{aligned}
$$

\section{References}

Abramovitz, M. and N. Stegun, 1970, Handbook of mathematical functions (Dover Publications, New York, NY)

Andersen, T.G., 1992, Volatility, Unpublished manuscript (Northwestern University, Evanston, IL).

Bollerslev, T., 1987, A conditional heteroskedastic time series model for speculative prices and rates of return, Review of Economics and Statistics 69, 542-547.

Bollerslev, T., R.Y. Chou, and K.F. Kroner, 1992, ARCH modeling in finance: A revicw of the theory and empirical evidence, Journal of Econometrics 52, 5-59.

Chesney, M. and L. Scott, 1989, Pricing European currency options: A comparison of the modified Black-Scholes model and a random variance model, Journal of Financial and Quantitative Analysis 24, 267-284.

Dunsmuir, W., 1979, A central limit theorem for parameter estimation in stationary time series and its applications to models for a signal observed white noise, Annals of Statistics 7, 490 506.

Engle, R.F, 1982, Autoregressive conditional heteroskedasticity with estimates of the variance of the U.K. inflation. Econometrica 50, 987-1007.

Hansen, L.P.. 1982, Large sample properties of generalized method of moments estimators, Econometrica 50, 1029-1054 
Harvey, A.C. 1989. Forecasting. structural models and the Kalman filter (Cambridge University Press. Cambridge).

Harvey, A.C., E. Ruiz, and N.G. Shephard. 1992, Multivariate stochastic variance model, Financial Markets Group discussion paper (London School of Economics. London).

Melino, A. and S.M. Turnbull, 1990, Pricing forcign currency options with stochastic volatility, Journal of Econometrics 45, 239-265.

Nelson, D.B., 1988, The time series behavior of stock market volatility and returns, Cnpublished Ph.D. dissertation (Massachusetts Institute of Technology. Cambridge. MA).

Taylor, S.J., 1991, Modeling stochastic volatility, Unpublished manuscript (I ancaster University, Lancaster).

Vetzal, K., 1991, Stochastic short rate volatility and the pricing of bonds and bond options, Unpublished Ph.D. dissertation (University of Toronto, Toronto). 